\title{
ESTUDAR E TRABALHAR: PERCEPÇÕES DE TÉCNICOS DE ENFERMAGEM SOBRE ESTA ESCOLHA
}

\author{
Study and work: perceptions of nursing technicians facing this choice
}

Estudiar y trabajar: percepciones de los técnicos de enfermería frente a esta elección

Rosane Teresinha Fontana'

Lariane Brigo $^{2}$

\section{RESUMO}

Pesquisa descritiva, de abordagem qualitativa, realizada com acadêmicos de enfermagem de uma universidade comunitária do Rio Grande do Sul/Brasil, no segundo semestre de 2009. Teve como objetivo identificar percepções de técnicos de enfermagem que vivenciam a experiência de trabalhar em um turno e estudar em outro. Os dados foram coletados por meio de um questionário e analisados pela metodologia de análise temática, resultando em quatro categorias: Concepções e percepções acerca da qualidade de vida; Situações que promovem qualidade de vida; Dificuldades encontradas durante o período acadêmico; Motivações à realidade de trabalhar e estudar. Embora com dupla jornada de atividades, para a maioria dos sujeitos é satisfatória sua qualidade de vida. 0 sono e a fadiga foram citados como as principais limitações para a dupla atividade plena. Discutir modos e meios de facilitar a conciliação trabalho e estudo é uma estratégia que pode agregar valor ao processo de formação destes sujeitos.

Palavras-chave: Enfermagem. Estudantes de Enfermagem. Qualidade de vida.

\begin{abstract}
Descriptive, qualitative study, conducted with nursing students from a university community of Rio Grande do Sul / Brazil in the second half of 2009 which aimed to identify perceptions of nursing technicians who experience working in one shift and in studying at the other. Data were collected through a questionnaire and analyzed using thematic analysis, resulting in four categories: Conceptions and perceptions about quality of life; Situations that promote quality of life; Difficulties encountered during the academic; Motivations to the reality of work and study. Although a double activity, for most subjects is satisfactory quality of life. Sleep and fatigue were cited as the main limitations for the dual full activity. Discuss ways and means of facilitating the conciliation work and study is strategies that can add value to the education of these subjects.
\end{abstract}

Keywords: Nursing. Students, Nursing. Quality of Life.

\section{Resumen}

Estudio descriptivo, cualitativo, realizado con estudiantes de enfermería de una comunidad universitaria en Río Grande do Sul / Brasil, en el segundo semestre de 2009. El objetivo de la investigación fue identificar las percepciones de los técnicos de enfermería que sufren la experiencia de trabajar en un turno y estudiar en otro. Los datos fueron recolectados y evaluados a través de un cuestionario, mediante análisis temático, resultando en cuatro categorías: Concepciones y Percepciones de la calidad de vida;Situaciones que promueven la calidad de vida; Dificultades encontradas durante la vida académica; Motivaciones para trabajar y estudiar. A pesar del doble turno de actividades, la mayoría considera satisfactoria la calidad de vida. El sueño y la fatiga son citados como los principales obstáculos para la plena actividad dual. Hablar acerca de formas y medios para facilitar la conciliación de trabajo y estudio es la estrategia que puede añadir valor a la formación de estos profesionales.

Palabras-clave: Enfermería. Estudiantes de Enfermería. Calidad de Vida.

\footnotetext{
Enfermeira. Mestre e Doutoranda pela EEUFRGS. Membro do GEPESE (Grupo de Estudos e Pesquisas em Enfermagem, Saúde e Educação). Professora da Universidade Regional Integrada do Alto Uruguai e das Missões. Santo Angelo-RS. Brasil. E-mail: rfontana@urisan.tche.br² Enfermeira da Conversora Garabi - Nordeste Emergência e Soluções Médicas Ltda - Pós-graduanda em Enfermagem do Trabalho do Sistema Educacional Galileu. São Luiz GonzagaRS. Brasil. E-mail: larianebrigo@hotmail.com
} 


\section{INTRODUÇÃO}

As exigências atuais estão for temente marcadas pelo crescimento da produtividade e inserção de novas tecnologias, situação que pode estar associada à busca pela qualificação profissional. Com isso, profissionais do nível médio da enfermagem estão buscando a graduação como um meio de crescimento pessoal, profissional e de conhecimentos.'

Em decorrência da situação socioeconômica da sociedade capitalista em que se vive atualmente, muitas pessoas precisam trabalhar para estudar a fim de conseguir pagar os cursos. ${ }^{1}$ Para estudar em escolas de graduação que oferecem o curso de enfermagem no turno diurno e integral, muitos trabalhadores, dispostos ao aperfeiçoamento, obrigamse a procurar serviços que thes proporcionem trabalhar em turnos, ou seja, trabalhar à noite para que seja possível estudar durante o dia, situação identificada neste estudo com técnicos de enfermagem.

A escolha pelo trabalho noturno inclui motivos que envolvem, entre outros, a remuneração diferenciada e a maior disponibilidade de tempo para a vida particular, ${ }^{2}$ o que beneficia o gerenciamento do cotidiano familiar e facilita a conciliação com atividades como outro emprego ou estudo.

Por outro lado, estudos que versam sobre o trabalho noturno apontam insatisfações relacionadas aos horários de trabalho coincidentes com feriados, finais de semanas, fragmentando, muitas vezes, os horários de lazer e as relações entre amigos e familiares, contribuindo, assim, para o isolamento social, além do sono e fadiga característicos da adaptação orgânica gerada pela alteração do ritmo biológico. ${ }^{2-3}$ Adstrito a isso, não se pode omitir que profissionais de enfermagem são predominantemente mulheres, com múltiplas funções, quais sejam de mãe, esposa e trabalhadora, condicionantes que podem comprometer a qualidade de vida destes sujeitos e configurar-se como fator de estresse.

Nossa indagação não se restringe aos problemas denunciados pela literatura acerca do trabalho noturno, ${ }^{2-3}$ abrange as consequências advindas desta sobrecarga de atividades do sujeito que trabalha em um turno e estuda em outro, considerando que tal configuração não implica somente a adaptação psicossocial, mas também fisiológica. Tem-se como exemplo a deficiência das horas de sono, situação que pode causar agravos à saúde deste trabalhador, considerando que, fisiologicamente, a noite é o momento em que o organismo se prepara para obter energia, alcançada pela diminuição natural das suas funções orgânicas.

Embora estudos comprovem que a percepção subjetiva do sono é maior em indivíduos mais jovens, a relação entre idade e sonolência é controversa. Uma pesquisa verificou que não há diferença entre idade e a sonolência diurna excessiva, porém é alta a prevalência destes episódios entre os trabalhadores da área da enfermagem, assim como do trabalho em turnos. ${ }^{4}$
A sonolência pode prejudicar o rendimento das atividades do trabalhador, como também os pacientes que necessitam de cuidado. Comparando-se trabalhadores submetidos a cargas horárias de 12 horas diurnas a outros com cargas horárias de 12 horas noturnas, seguidas de 36 horas de descanso, concluiuse que, nos últimos, a qualidade do sono foi pior, ${ }^{5}$ o que pode causar prejuízo tanto aos trabalhadores quanto aos pacientes que estão sob seus cuidados; o sono do dia apresenta-se fragmentado em decorrência de várias especificidades, como a necessidade de realizar tarefas domésticas ou até mesmo pela própria dificuldade de adaptação da alternância de turnos, além de que, durante o dia, há barulho decorrente do trânsito, do movimento da casa, entre outros.

Neste sentido, entende-se que a qualidade de vida e saúde do trabalhador estudante está ameaçada, tendo em vista que, não bastasse a exposição ao sofrimento, a dor e a morte, presentes em seu processo de trabalho, a sobrecarga de atividades que se estabelece entre estudo e trabalho e as alterações fisiológicas características da adaptação constante ao novo ritmo instalado podem ser fatores de desgaste físico e mental.

Sendo a enfermagem uma profissão comprometida com o cuidado e melhoria da qualidade de vida do outro, é fundamental que o profissional disponha, também, de boas condições de vida e trabalho. Não se trata de coibir a decisão ou a necessidade do aluno de trabalhar e estudar, mas conhecer a realidade para propor alternativas de promoção à saúde deste sujeito.

Empiricamente, observa-se que a trajetória acadêmica é uma etapa que se caracteriza por momentos promotores de prazer e de desprazer, considerando-se que é um espaço para a aquisição de novos amigos, de novas esperanças, mas também de exigências cognitivas implicadas neste contexto, o que, por si só, pode interferir no curso da qualidade de vida do aluno.

Em um estudo que buscou compreender como os graduandos de enfermagem percebem a universidade como espaço promotor de qualidade de vida, os resultados demonstrados revelam que ora ela é espaço promotor de qualidade de vida, ora não é. 0 aprendizado adquirido, a cidadania respeitada e o relacionamento interpessoal promovem alegrias aos estudantes e qualificam a vida, ao contrário das angústias, inseguranças e medos, principalmente nos estágios, e das relações conflituosas com professores, que a desqualificam. Outro aspecto considerado como não promotor de qualidade de vida foi relacionado com a excessiva carga horária do curso e com a situação desgastante gerada pelo cotidiano estudotrabalho. ${ }^{6}$

Buscando compreender como o trabalho e o estudo, realizados simultaneamente, podem interferir na vida do estudante é que esta pesquisa se justifica. Sendo assim, o estudo teve como questão norteadora: Como é vivenciada a experiência de trabalhar e estudar e qual a interface dessa situação na qualidade de vida desses sujeitos? 
A pesquisa teve como objetivo identificar percepções, quanto à qualidade de vida, de técnicos em enfermagem que vivenciam a experiência de trabalhar e estudar.

\section{METODOLOGIA}

Trata-se de um estudo descritivo, de abordagem qualitativa. A coleta de dados foi realizada com 25 acadêmicos de enfermagem de uma universidade comunitária situada na região noroeste do estado do Rio Grande do Sul/Brasil, durante o primeiro semestre de 2010, mediante a aplicação de um questionário com perguntas abertas. 0 critério utilizado para delimitar este quantitativo foi convidar a todos os acadêmicos, dos quatro semestres em curso $\left(3^{\circ}, 5^{\circ}, 7^{\circ} \mathrm{e} 9^{\circ}\right)$, à participação do estudo, do qual resultou esta população.

Sendo assim, foram critérios para participar do estudo: ser acadêmico regular do curso de enfermagem de qualquer semestre, estar trabalhando como profissional técnico em enfermagem em instituição de saúde e/ou no cuidado ao usuário/paciente. Excluíram-se os trabalhadores de outros setores da economia.

0 estudo não teve a intenção de associar variáveis ou classificar os sujeitos quanto ao período do curso em que se encontravam, mas refletir acerca da qualidade de vida do acadêmico-trabalhador independente de fatores circunscritos.

0 questionário autoaplicável, composto por oito questões, que versaram sobre concepções relacionadas à vivência do sujeito quanto a trabalhar e estudar, motivações e dificuldades, ações promotoras e não promotoras de qualidade de vida, foi entregue durante o período de aulas teóricas e respondidos no mesmo momento.

A análise dos dados realizou-se mediante análise de conteúdo das falas, na modalidade análise temática. ${ }^{7}$ Os participantes foram identificados por nomes de flores, garantindo seu anonimato. 0 estudo obteve parecer favorável do Comitê de Ética da Universidade Regional Integrada do Alto Uruguai e das Missões/Campus Santo Ângelo/RS, sob número de protocolo 096-4/PPH/09, bem como autorização/ciência da coordenação do curso de enfermagem da universidade pesquisada e assinatura do Termo de Consentimento Livre e Esclarecido pelos sujeitos, de acordo com a Resolução do Conselho Nacional de Saúde nº. 196/96.

\section{RESULTADOS E DISCUSSÃO}

Participaram do estudo 25 acadêmicos, de 22 a 42 anos, sendo prevalente a idade compreendida entre 22 e 27 anos, representando $64 \%$ dos estudantes dos $3^{\circ}, 5^{\circ}, 7^{\circ}$ e $9^{\circ}$ semestres do Curso de Enfermagem de uma universidade regional. 0 sexo feminino representou $68 \%$ da amostra. Todos os sujeitos trabalham em instituições de saúde do município em que se localiza o curso de graduação em enfermagem.

\section{Concepções e percepções acerca da qualidade de vida}

Quando questionados sobre suas concepções acerca de qualidade de vida, observou-se que as respostas, semelhante a outra realidade pesquisada ${ }^{6}$, aproximaram-se do conceito de saúde preconizado pela lei 8080 de $1990^{\circ}$, a qual condiciona a saúde a fatores como a alimentação, moradia, saneamento básico, o meio ambiente, o trabalho, a educação, o lazer, entre outros.

Qualidade de vida é viver bem, trabalhar na área que gosto, ter um bom relacionamento familiar e um equilibrio entre lazer e trabalho. (Violeta)

Possuir trabalho fixo, ter saúde, ter tempo para a minha familia, para descansar, boa alimentação e um sono tranquilo. (Bromélia)

É integrar lazer, trabalho, familia, crescimento profissional e a autoestima de forma harmoniosa, sem sobrecarregar cada etapa. (Orquídea)

É conseguir conciliar os estudos, o trabalho, a casa, familia, é ter saúde, conforto, hábitos saudáveis e momentos de lazer. (Hortência)

Embora com dupla jornada de atividades, a maioria dos sujeitos avalia como satisfatória sua condições de vida e saúde, independente da distribuição do tempo, pois relatam saúde, situação financeira estável e admitem conseguir conciliar a profissão com os estudos e tarefas familiares, como é possível visualizar nas respostas a seguir:

Sim [tenho qualidade de vida], pois tenho saúde, realizo atividades físicas, tenho um emprego no qual desempenho atividades que gosto, amo minha profissão, minha família e sempre procuro manter um bom relacionamento entre colegas. (Girassol)

Sim, estou satisfeita com a profissão que escolhi, a empresa que trabalho me valoriza e me proporciona aprimorar novos conhecimentos. (Violeta)

Sim, vivo com as pessoas que amo, trabalho para pagar meus estudos e mesmo assim ainda consigo achar um tempo para brincar com meu filho e realizar atividades de lazer com minha família. (Cravo)

Não foi intenção deste estudo verificar a relação entre condições de vida e saúde e o período ou semestre letivo em que 0 acadêmico se encontrava no curso; porém, acredita-se que a qualidade de vida dos acadêmicos de enfermagem veteranos, ou seja, a partir do segundo ano, é menor do que a dos ingressantes na universidade. Isso pode ser justificado pela inserção do aluno em campo prático, característica do quarto 
semestre no cenário estudado, fator gerador de conflitos e mudanças no cotidiano acadêmico, considerando que é uma ocasião de novas experiências e novos sentimentos, tais como insegurança, medo e ansiedade ${ }^{6,9}$ gerados com o novo, a avaliação, a aceitação do paciente e dos trabalhadores do campo prático, entre outros.

\section{Situações que promovem qualidade de vida}

Entre as situações que promovem qualidade de vida para os entrevistados, foram relatadas que viajar, sair para se divertir com os amigos e/ou família, ter uma noite de sono tranquila, ter uma situação financeira estável, entre outras, influenciam na qualidade de vida dos sujeitos. Quando se trata de qualidade de vida, os sujeitos logo a associam a lazer, descanso, diversão.

O que promove qualidade e vida para mim é estar em um bom estado de saúde, situação financeira estável, ter a presença de meus amigos ao meu lado e poder ter momentos de lazer. (Bromélia)

Estudar, sair para me divertir, descansar, praticar atividades físicas e ver um bom filme. (Violeta)

Estar saudável, poder trabalhar, viajar, ter uma alimentação saudável, ler um bom livro, sair para dançar e conversar com os amigos, mas principalmente ter uma boa noite de sono. (Vitóriarégia)

Com o objetivo de identificar a frequência de lazer vivenciada pelos universitários, em Rio Claro/SP, e se havia diferenças entre o lazer dos estudantes trabalhadores e dos não trabalhadores, uma pesquisa ${ }^{10}$ demonstrou que, assim como na realidade local, praticar atividades de lazer não é uma ocorrência comum; se restringe a uma a duas vezes por semana, normalmente nos finais de semana, provavelmente quando não há a necessidade de estudar para provas e realizar trabalhos, ou mesmo trabalhar em plantões. A fadiga aparece como a única causa para a restrição do lazer pelos trabalhadores.

\section{Dificuldades encontradas durante 0 período acadêmico e qualidade de vida}

Em se tratando das principais dificuldades encontradas pelos acadêmicos trabalhadores, a sonolência diurna é prevalente. A fadiga, segunda dificuldade citada, vem a ser uma consequência da falta de tempo para dormir. Em decorrência destes fatores, manter a concentração durante o período de aula e estágios pode configurar-se como uma dificuldade aos sujeitos e prejudicar o aprendizado.

Porém, há divergências acerca desta situação. Em uma avaliação da percepção do sono e da fadiga, comparandose trabalhadores de enfermagem de turnos diferentes, foi demonstrado que, mesmo trabalhando em dois empregos e em turnos diferentes, isso não se mostrou significativo para a fadiga na rotina do trabalhador. Nesta pesquisa, a fadiga não se mostrou significante em trabalhadores com mais de 10 anos na profissão e que trabalham em turno noturno, assim como entre aqueles trabalhadores com dois empregos; ao contrário, profissionais que trabalham em um único turno diurno e com menos tempo na carreira mostraram-se mais fatigados. ${ }^{11}$

Entretanto, pode-se inferir que estudar e trabalhar pode ter outro significado, considerando que além da carga horária destinada às aulas teórico-práticas tem-se, ainda, a rotina extraclasse compreendida pelos trabalhos acadêmicos, estudos e atividades extracurriculares. Alguns sujeitos relatam a falta de qualidade de vida em virtude das múltiplas tarefas e da indisponibilidade de tempo para descanso e ou lazer.

Não tenho qualidade de vida, porque estudo de dia, trabalho de noite. Como consequência não consigo dormir as horas necessárias para o bom funcionamento do meu organismo, não tenho horários certos para me alimentar, fazer exercícios e tenho poucas horas para o meu lazer. (Vitóriarégia)

Falta qualidade de vida em decorrência do pouco tempo livre que tenho para realizar exercícios físicos; meu tempo com a familia é racionado. Não, pois meu sono é excessivo, tenho uma má alimentação e uma sobrecarga de tarefas (trabalho, estágios, aula, provas, família e lazer). (Copo-deleite)

Pode-se inferir que o sedentarismo e os hábitos alimentares incorretos podem estar relacionados com as modificações que ocorrem na vida acadêmica e ocupacional. 0 trabalhador, mesmo cansado do cotidiano acadêmico, precisa trabalhar, e vice-versa. Fatigado, não tem ânimo para praticar atividade física e, em virtude do tempo escasso, alimenta-se mal, seja em qualidade ou em quantidade.

Abuso na má alimentação comendo apenas lanches para conseguir realizar outras tarefas no momento em que eu estaria cozinhando. (Lírio)

0 sedentarismo está presente na vida de muitos universitários, especialmente na população feminina e em estudantes do período noturno, e pode estar relacionado à falta de tempo e de motivação para a prática de exercícios físicos, ${ }^{12}$ o que preocupa, considerando que é um fator de risco significativo para um possível desenvolvimento de doença arterial coronariana. ${ }^{13}$

Sendo assim, o estudante é um sujeito vulnerável ao adoecimento em decorrência do trabalho, considerando que se 
expõe constantemente a jornadas duplas pela prática de estágios e aulas, concomitantes com suas atividades laborais e familiares. Um estudo realizado nas Filipinas ${ }^{14}$ com 655 enfermeiros identificou que o trabalho em turnos e horas-extras pode estar associado a acidentes e a doenças, além de absenteísmo e/ou afastamentos por licença-saúde

Isto posto, situações de estresse também podem estar presentes no decorrer do curso de graduação, independentemente do período de formação em que os estudantes estão matriculados. Além do estresse por conta do vestibular, cursar determinadas disciplinas que exigem práticas laboratoriais nunca antes realizadas e cumprir uma carga horária semanal distribuída em mais de um turno são fatores estressores que se refletem na qualidade da sua saúde mental, tornando-os mais irritados, ansiosos, com baixa autoestima e desestimulados. ${ }^{15}$

0 estresse é um agravo comum no cotidiano da enfermagem. Estudos demonstram que a prevalência de sofrimento mental é comum entre os profissionais e condições de trabalho adversas podem contribuir para estes agravos, tais como condições inadequadas do ambiente físico, sobrecarga física e psicológica, tempo de horas de trabalho igual ou superior a 10 horas por dia e trabalho em turnos (o que implica ter menos tempo para os filhos e para a vida familiar), violência física e verbal, exigências por parte dos usuários/pacientes e seus familiares, irregularidades e/ou problemas alimentares, ${ }^{16-}$ ${ }^{18}$ estes últimos reforçados pelo consumo excessivo de chá e café durante a trabalho noturno, as dificuldades na administração do tempo para as refeições, entre outras. ${ }^{17}$

\section{Motivações que movem a realidade de trabalhar e estudar}

Com relação aos motivos pelos quais os entrevistados voltaram a estudar, o possível aumento da remuneração financeira e a aquisição de conhecimentos são fatores que influenciaram na tomada desta decisão, como pode ser observado nas falas seguintes:

A remuneração financeira, que no futuro irá aumentar. Consequentemente poderei trabalhar menos e me dedicar mais à família, além do conhecimento adquirido. (Tulipa)

Adquirir novos conhecimentos, melhorar a qualidade de vida profissional (principalmente com relação ao salário), lutar e acreditar em uma enfermagem mais humanizada. (Vitória-régia)

Maior remuneração financeira, o crescimento pessoal e a busca pelo conhecimento. (Flor-docampo)
Um estudo que teve o objetivo de levantar as expectativas dos acadêmicos de enfermagem, com relação ao futuro mercado profissional ${ }^{19}$, encontrou resultados semelhantes aos achados nessa pesquisa, uma vez que mais da metade dos entrevistados pretendem receber salários mensais acima de quatro salários mínimos.

Relevante, também, é que os sujeitos acreditam que devem cursar uma pós-graduação subsequente à graduação, tendo em vista que as exigências para o profissional recémformado estão aumentando e que o mercado de trabalho está cada vez mais seletivo.

\section{CONSIDERAÇÕES FINAIS}

Esta pesquisa revelou que estudantes-trabalhadores de enfermagem reconhecem o trabalho noturno como desgastante, mas, ainda assim, tentam conviver com esta situação a fim de conseguirem manter os estudos. As principais dificuldades encontradas para conciliar trabalho e estudo, segundo os sujeitos, são a fadiga e o sono.

Observou-se que não só motivados pelo saber é que os trabalhadores buscam a graduação, mas também pelo desejo de melhores condições de trabalho e salários, pois, na medida em que evoluem na construção do conhecimento, têm perspectivas de mudança do padrão financeiro, o que demonstra coragem e determinação na busca de seus objetivos, considerando-se que é árdua a batalha de estudar e trabalhar.

Assim, a partir das dificuldades específicas, podem ser alternativas para estes trabalhadores cursar disciplinas oferecidas na modalidade de ensino à distância, que são comuns a outros cursos, e portanto em horários flexíveis, além de habituar-se ao processo de organização do trabalho e estudo para que, com saúde, consigam vencer esta etapa.

Além disso, é fundamental que gestores e enfermeiros se sensibilizem com esta situação, a fim de que, com compreensão, contribuam para a consolidação dos sonhos da formação superior destes trabalhadores. 0 contexto de saúde só tem a ganhar com os estudantes, pois são sujeitos que, com a mesma remuneração, contribuem com saberes e práticas atualizadas para a qualidade do processo do cuidado, seja por meio da educação, da pesquisa ou da extensão.

0 estudo não encontrou limitações à sua realização. É válido salientar que não houve a intenção de generalizar os achados, pois são peculiares à realidade estudada.

Sugerem-se novas pesquisas sobre esse tema, visando revisar e aperfeiçoar o acesso destes profissionais à graduação, bem como discutir modos e meios de facilitar o vínculo trabalho e estudo, assim como investigar a qualidade de vida dos estudantes trabalhadores comparando-se os escores de antes e depois do início dos estágios. 


\section{REFERÊNCIAS}

1.Medina NVJ, Takahashi RT. A busca da graduação em enfermagem como opção dos técnicos e auxiliares de enfermagem. Rev Esc Enferm USP. 2003; 37(4):101-08.

2. Silva RM, Beck CLC, Guido LA, Lautert L, Tavares JP, Prestes FC, Rocha L. Night shift pros and cons in nursing: qualitative study. Online Braz. J. Nurs. [on-line] 2009 [citado 2010 maio 12]; 8(2): [aprox. 5 telas]. Disponível em: http://www.objnursing.uff.br/index.php/nursing/article/view/2346

3. Lisboa MTL, Oliveira MM, Reis LD. 0 trabalho noturno e a prática de enfermagem: uma percepção dos estudantes de enfermagem. Esc Anna Nery. 2006; 10(3): 393-98

4. Souza JC. Sonolência diurna excessiva em trabalhadores da área de enfermagem. J Bras Psiquiatr. 2007; 56(3): 180-83.

5. Fischer FM, Teixeira LR, Borges FNS, Gonçalves MBL, Ferreira RM. Percepção de sono: duração, qualidade e alertas em profissionais da área de enfermagem. Cad Saude Publica. 2002; 18(5): 1261-69

6. Oliveira RA, Ciampone MHT. A universidade como espaço promotor de qualidade de vida: vivencias e expressões dos alunos de enfermagem. Texto\&Contexto Enferm. 2006 abr/jun; 15(2): 254-61

7. Minayo MCS. 0 desafio do conhecimento: pesquisa qualitativa em saúde. $7^{\mathrm{a}}$ ed. Rio de Janeiro: Abrasco; 2008.

8. Conselho Nacional de Saúde (BR) [on-line]. Lei n 8080, de 19 de setembro de 1990. Dispõe sobre as condições para promoção, proteção e recuperação da saúde, a organização e o funcionamento dos serviços correspondentes e dá outras providências [aprox. 4 telas]; 1990 [citado 2010 mai0 14]. Disponivel em: http://conselho.saude.gov.br/legislacao/ lei8080_190990.htm

9. Bosquetti LS, Braga EM. Reações comunicativas dos alunos de enfermagem frente ao primeiro estágio curricular. Rev Esc Enferm USP. 2008 ; 42(4): 690-96.

10. Martoni FR, Schwartz GM. 0 lazer na vida do estudante universitário. Rev Digital [on-line] 2006 [citado 2010 jun 3] [aprox 4 telas]. Disponível em: http://www.efdeportes.com/efd97/lazer.htm

11. Rosa PLFS, Fischer FM, Borges FNS, Soares NS, Rotenberg L, Landsbergis P. Percepção da duração do sono e da fadiga entre trabalhadores de enfermagem. Rev Enferm UERJ. 2007; 15(1): 100-06.

12. Quadros TMB, Petroski EL, Silva DAS, Gordia AP. Prevalência de sedentarismo em universitários brasileiros: associação com variáveis sociodemográficas. Rev Saude Publica. 2009; 11(5): 724-33.

13. Alves A, Marques IR. Fatores relacionados ao risco de doença arterial coronariana entre estudantes de enfermagem. Rev Bras Enferm. 2009; 62(6): 883-88.

14. Castro AB, Fujishiro K, Rue T, Tagalog EA, Samaco-Paquiz LPG, Gee GC. Associations between work schedule characteristics and occupational injury and illness. Int Nurs Rev. 2010 June; 57(2): 188-94.
15. Monteiro CFS, Freitas JFM, Ribeiro AAP. Estresse no cotidiano acadêmico: o olhar dos alunos de enfermagem da Universidade Federal do Piauí. Esc Anna Nery. 2007 mar; 11(1): 66-72

16. Malinauskienë V, Leiđytë P, Malinauskas R. Psychosocial job characteristics, social support, and sense of coherence as determinants of mental health among nurses. Medicina (Kaunas) [on-line] 2009 [cited 2010 dez 20; 45(11):10-17. Available from: http://medicina.kmu.tt/0911/ 0911-09e.htm

17. Kane PP. Stress causing psychosomatic illness among nurses Indian 」 Occup Environ Med. [on-line] 2009 Apr; [cited 2010 dez 20] 13(1): 2832. Available from: http://www.ncbi.nlm.nih.gov/pmc/articles/ PMC2822165/?tool=pubmed

18. Wu S, Li H, Tian J, Zhu W, Li J, Wang X. Health-related quality of life and its main related factors among nurses in China. Ind Health. [on-line] 2011; [cited 2010 dez 20]; 49(2):158-65. Available from: http:// uww.ncbi.nlm.nih.gov/pubmed

19.Manarin AP, Bortolete CB, Ferreira-Sae MCS. Perspectivas do egresso de enfermagem frente ao mercado de trabalho. Rev Ensaios e Ciencias [on-line] 2009 [citado 2010 jun 17]; 9 (1): 93-105. Disponível em: http:/ /redalyc.uaemex.mx/redalyc/pdf/260/26012800009.pdf 